\title{
Sialyllactose suppresses angiogenesis by inhibiting VEGFR-2 activation, and tumor progression
}

\author{
Tae-Wook Chung ${ }^{1}$, Eun-Young Kim ${ }^{1,2}$, Seok-Jo Kim ${ }^{3}$, Hee-Jung Choi ${ }^{1}$, Se Bok Jang ${ }^{4}$, \\ Keuk-Jun Kim ${ }^{5}$, Sun-Hyung Ha ${ }^{3}$, Fukushi Abekura ${ }^{3}$, Choong-Hwan Kwak $^{3}$, Cheorl- \\ Ho $\mathrm{Kim}^{3}$ and Ki-Tae Ha ${ }^{1,2}$ \\ ${ }^{1}$ School of Korean Medicine, Healthy Aging Korean Medical Research Center, Pusan National University, Yangsan, \\ Gyeongsangnam-do 50612, Republic of Korea \\ ${ }^{2}$ Graduate Training Program of Korean Medicine for Healthy-Aging, Pusan National University, Yangsan, Gyeongsangnam-do \\ 50612, Republic of Korea \\ ${ }^{3}$ Department of Biological Science, Sungkyunkwan University, Suwon, Kyunggi-do 16419, Republic of Korea \\ ${ }^{4}$ Department of Molecular Biology, College of Natural Sciences, Pusan National University, Geumjeong-gu, Busan 46241, \\ Republic of Korea \\ ${ }^{5}$ Department of Clinical Pathology, TaeKyeung University, Gyeongsan 38547, Republic of Korea \\ Correspondence to: Cheorl-Ho Kim, email: chkimbio@skku.edu \\ Ki-Tae Ha, email: hagis@pusan.ac.kr \\ Keywords: sialyllactose, milk, oligosaccharides, angiogenesis, VEGF receptor-2 \\ Received: May 31, $2016 \quad$ Accepted: February 15, $2017 \quad$ Published: March 14, 2017 \\ Copyright: Chung et al. This is an open-access article distributed under the terms of the Creative Commons Attribution License \\ 3.0 (CC BY 3.0), which permits unrestricted use, distribution, and reproduction in any medium, provided the original author and \\ source are credited.
}

\section{ABSTRACT}

The oligosaccharides in human milk have various biological functions. However, the molecular mechanism(s) underlying the anti-angiogenic action of sialylated human milk oligosaccharides (HMOs) are still unclear. Here, we show that siallylactose (SL) found in human milk can inhibit the activation of vascular endothelial growth factor (VEGF)mediated VEGF receptor-2 (VEGFR-2) by binding to its VEGF binding site (second and third IgG-like domains), thus blocking downstream signal activation. SL also inhibits growth of VEGF-stimulated endothelial cells. In endothelial cells treated with VEGF, SL diminished tube formation, migration, and the arrangement of actin filament. In addition, SL clearly suppressed VEGF-induced neovascularization in an in vivo Matrigel plug assay. Notably, SL prevented the growth of tumor cells, and angiogenesis on tumor tissues in in vivo mice models allotransplanted with Lewis lung carcinoma, melanoma, and colon carcinoma cells. Taken together, we have demonstrated that the sialylated milk oligosaccharide sialyllactose functions as an inhibitor of angiogenesis through suppression of VEGFmediated VEGFR-2 activation in endothelial cells, Accordingly, it could be a novel candidate for the development of anti-angiogenic drugs without any side effects.

\section{INTRODUCTION}

Human milk is composed of many bioactive ingredients, including milk oligosaccharides [1, 2]. It has been suggested that human milk oligosaccharides (HMOs) have diverse biological functions. They can function as including prebiotics, anti-microbials, modulators of intestinal epithelial cell, and regulators of the immune system [3, 4]. HMOs form a complex group of oligosaccharides composed of more than a hundred different glycans. They are constructed by five basic monosaccharide elements, including glucose (Glc), galactose (Gal), $N$-acetylglucosamine (GlcNAc), fucose (Fuc), and sialic acid (Sia) [4]. HMOs are contained of lactose on the reducing end, a polylactosamine core, and often fucose or sialic acid at the nonreducing terminus [5, 6]. Among them, sialylated HMOs have unique biological functions, such as inhibition of cell-cell interaction and pathogen-host interaction, because of their negative charge and hydrophilic nature $[3,7,8]$. Cell surfaced glycans, especially sialic acid-containing oligosaccharides, play critical roles in the progression of tumorigenesis, including 
neoplastic transformation, survival, tumor-induced immune modulation, and metastasis [9]. Acetamido GM3 (a-GM3 including lactose moiety with sialic acid) which forms the core glycan component of the gangliosides, interacts with human galectin-3 associated with diseases including cancer, inflammation, and angiogenesis [10].

Several studies have demonstrated that HMOs have a suppressive effect on carcinogenesis and tumor growth $[11,12]$. Furthermore, Rudolff et al. [13] demonstrated that sialylated HMOs have anti-angiogenic activity. However, the exact composition of oligosaccharides and underlying molecular mechanism responsible for the antiangiogenic action of sialylated HMOs are still unclear.

In this study, we showed sialyllactose, a basic unit of sialiylated HMOs, has a suppressive effect on the phosphorylation of VEGFR-2 in endothelial cells through direct binding to the extracellular domain of the receptor. Sialyllactose inhibits angiogenesis in vitro and in vivo, and consequently reduces the growth of allograft tumors. These findings indicate that sialyllactose can be a novel anti-angiogenic drugs candidate.

\section{RESULTS}

\section{Sialyllactose suppresses VEGFR-2 phosphorylation in HUVECs}

Although more than 150 oligosaccharides exist in HMOs, there are several structural core units, such as lactose, lacto $N$-biose, $N$-acetyllactosamine, 3 '-sialyl$N$-lactosamine $\quad(3 \mathrm{SNL}), \quad 6^{\prime}$-sialyl- $N$-lactosamine (6SNL), 3'-sialyllactose (3SL), and 6'-sialyllactose (6SL) (Supplementary Figure 1) [2, 4]. As shown in (Supplementary Figure 1), basic HMOs did not affect on the cytotoxicity of HUVECs. However, among the basic HMOs, 3SL and 6SL have a dose-dependent inhibitory effect on the phosphorylation of VEGFR-2 in VEGFstimulated HUVECs (Figure $1 \mathrm{~F}$ and $1 \mathrm{G}$ ). As shown in (Figure 1), 3SL and 6SL, composed of sialic acid, galactose, and glucose, both inhibited VEGF-induced VEGFR-2 activation. Accordingly, we used 3SL in subsequent experiments.

\section{Sialyllactose inhibits VEGF-induced growth, tube formation, migration and formation of actin stress fibers in HUVECs}

Although sialyllactose did not induce cytotoxicity of HUVECs (Supplementary Figure $1 \mathrm{~F}$ and $1 \mathrm{G}$ ), 3SL inhibited VEGF-stimulated growth of HUVECs in a dose-dependent manner (Figure 2A and Supplementary Figure 2). In addition to its inhibitory effect on the phosphorylation of VEGFR-2, 3SL suppressed the activation of signaling pathways downstream of VEGFR-2, including ERK, Akt, and p38. These pathways are related to proliferation, migration, formation of actin filaments and tube formation of VEGF-induced HUVECs (Figure 2B). The capillary-like tube formation of HUVECs

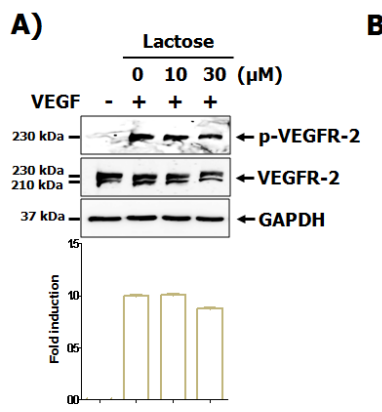

D)

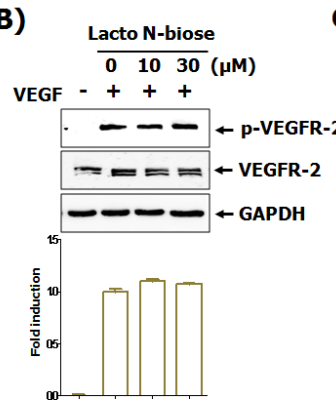

E)

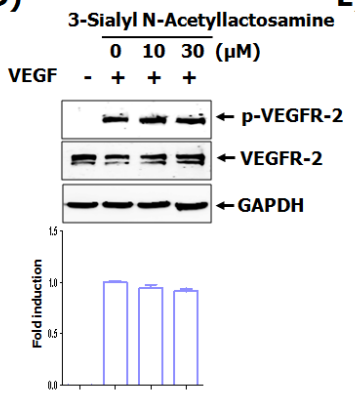

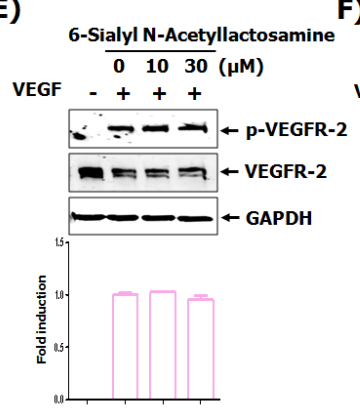

C)

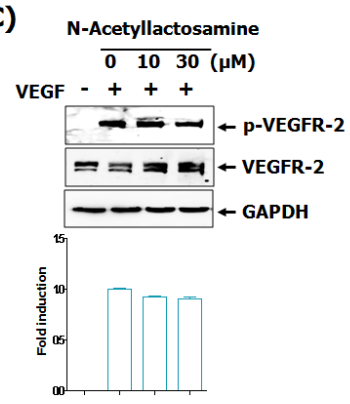

F)
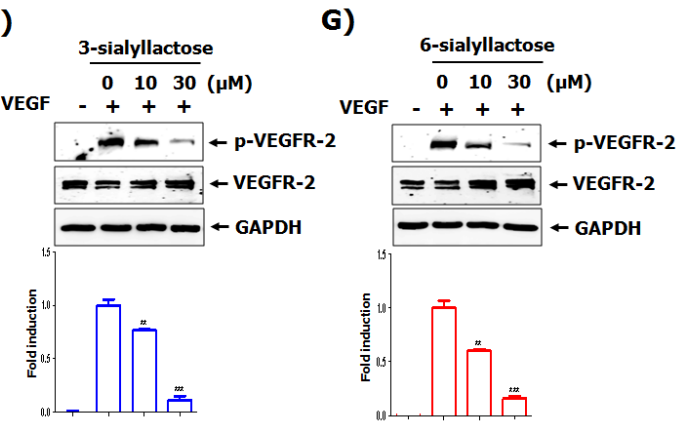

Figure 1: Screening of milk sialic oligosaccharides for their ability to inhibit VEGFR-2 activation. (A-G) HUVECs were starved in EBM-2 containing $1 \%$ FBS for $6 \mathrm{~h}$. The cells were pretreated with indicated concentrations of each oligosaccharide for $1 \mathrm{~h}$ and stimulated with VEGF ( $50 \mathrm{ng} / \mathrm{mL}$ ) for $15 \mathrm{~min}$. The phosphorylation of VEGFR-2 was determined by western blot analysis. Densitometric analysis was performed and the relative value of p-VEGFR-2/VEGFR-2/GAPDH is shown as fold change compared to the positive control. $* * p<0.01$ and $* * * p<0.001$ compared to positive control ( $2^{\text {nd }}$ lane $)$. 
induced by VEGF was also reduced by $3 \mathrm{SL}$ treatment (Figure 3A). Furthermore, VEGF-stimulated migration of HUVECs was remarkably decreased with 3SL treatment (Figure 3B). 3SL also inhibited VEGF-induced migration of HUVECs, as determined by wound healing assay (Figure 3C). Moreover, 3SL effectively suppressed VEGFinduced marked formation of actin filaments in HUVECs, and inhibited VEGF-stimulated the recruitment of paxillin to filamentous arrays, indicating contribution to cell migration (Figure 3D).

\section{Sialyllactose directly binds to extracellular domain (ExD) of VEGFR-2}

By immunoprecipitation and high performance thin layer chromatography (HPTLC), we examined whether the inhibitory effect of 3SL on VEGFR-2 phosphorylation is due to direct interaction with the ExD of VEGFR-2 (Figure 4A). The results clearly demonstrated that $3 \mathrm{SL}$ interacted with the ExD of VEGFR-2 (Figure 4B). Next, we modeled the interaction

A)

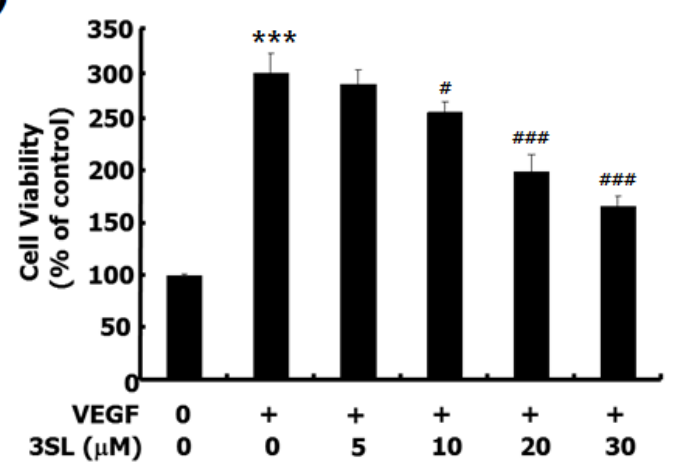

B)

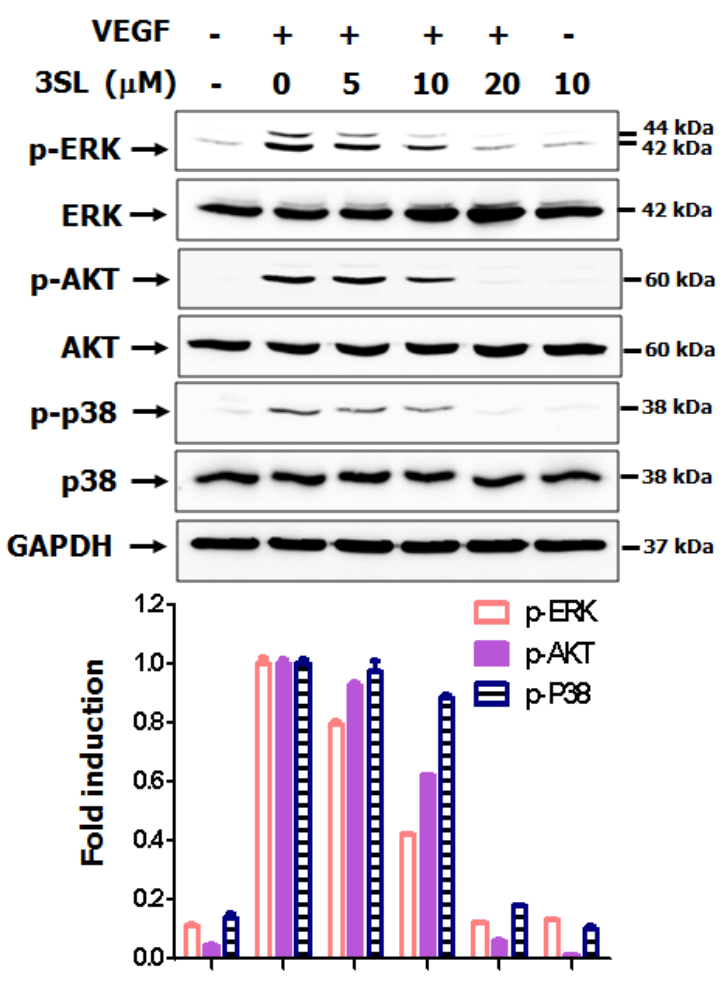

Figure 2: Effects of sialyllactose on the viability of HUVECs and activation of signal pathways stimulated by VEGF. (A) HUVECs were treated with the indicated concentrations of sialyllactose in the presence or absence of VEGF (50 ng/mL) for $72 \mathrm{~h}$. The effect of sialyllactose on the viability of VEGF-treated HUVECs was measured by MTT assay and are presented as means \pm SD. *** $p<0.001$ compared to control ( $1^{\text {st }} l$ lane), \# $p<0.05$ and \#\#\# $p<0.001$ compared to the positive control ( $2^{\text {nd }}$ lane). (B) The HUVECs were starved in EBM-2 containing $1 \%$ FBS for $6 \mathrm{~h}$. The cells were pretreated with indicated concentrations of each oligosaccharide for $1 \mathrm{~h}$ and stimulated with VEGF $(50 \mathrm{ng} / \mathrm{mL})$ for $15 \mathrm{~min}$. The phosphorylation of ERK, AKT, and p38 was analyzed by western blot analysis. Densitometric analysis was performed and the relative value of p-ERK/ERK/GAPDH, p-AKT/AKT/GAPDH, and p-p38/p38/GAPDH is shown as fold change relative to the positive control. 
of 3SL with the ExD of VEGFR-2 (PDB ID: 3S35), following a protein-small molecule docking method (Figures 4C-4G). Notably, 3SL bound to the purified second and third IgG-like domains of VEGFR-2, as validated by Biacore assay, but $3 \mathrm{SNL}$ did not (Figure $4 \mathrm{H}$ and 4I).

\section{Sialyllactose inhibits angiogenesis and tumor growth in vivo}

To evaluate the in vivo effectiveness of sialyllactose on angiogenesis, a Matrigel plug assay was performed. The results showed that VEGF-stimulated vascularization

A)
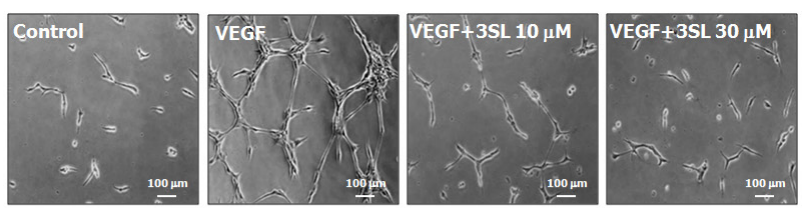

B)
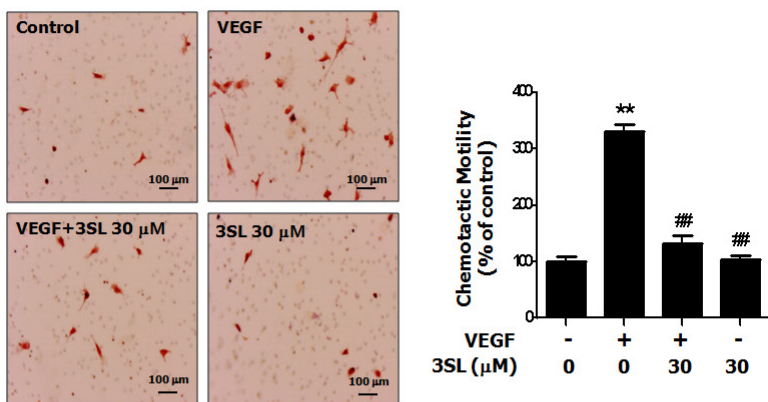

C) Control
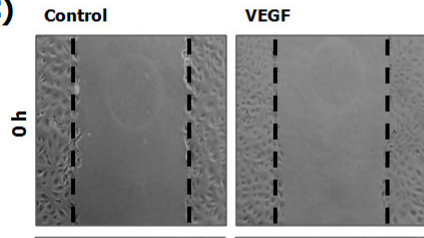

VEGF+3SL $30 \mu \mathrm{M}$
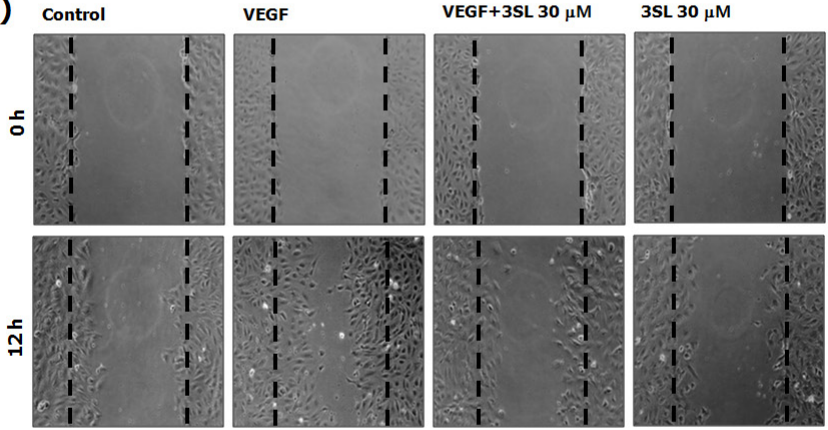

D) control
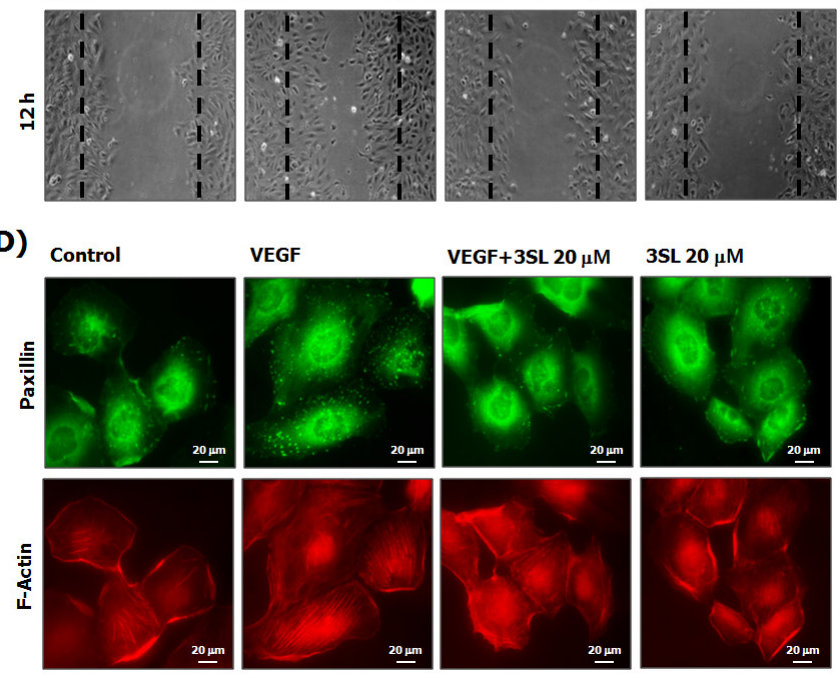

Figure 3: Suppressive effects of sialyllactose on tube formation, migration and formation of the actin stress fibers of HUVECs. (A) HUVECs cultured on matrigel coated plates were treated with the indicated concentrations of sialyllactose and stimulated with VEGF $(50 \mathrm{ng} / \mathrm{mL}$ ) for $15 \mathrm{~h}$. Morphological changes in HUVECs were observed by microscopy and representative images are shown. (B) HUVECs were seeded in the upper part of the Boyden chamber and treated with the indicated concentrations of sialyllactose in upper chamber. VEGF $(50 \mathrm{ng} / \mathrm{mL})$ was added to the lower compartment of the chamber. The chambers were incubated at $37^{\circ} \mathrm{C}$ for $24 \mathrm{~h}$ in a $5 \%$ $\mathrm{CO}_{2}$ atmosphere. The migrated cells stained with hematoxylin and eosin were imaged and counted. The numbers of migrated HUVECs are shown as the means $\pm \mathrm{SD} .{ }^{* *} p<0.01$ compared to the control ( $\left(^{\text {st }}\right.$ lane $)$ and $\# \# p<0.01$ compared to the positive control $\left(2^{\text {nd }}\right.$ lane $)$. (C) The HUVECs were confluently grown. Monolayers of HUVECs were wounded. The cells were treated with the indicated concentrations of sialyllactose and stimulated with VEGF $(50 \mathrm{ng} / \mathrm{mL}$ ) for $12 \mathrm{~h}$. (D) The formation of HUVEC actin stress fibers was analyzed by staining paxillin and F-actin using a specific antibody and phalloidin, respectively. 
was markedly suppressed by 3SL treatment (Figure 5). In addition, the effect of $3 \mathrm{SL}$ on the growth of the allograft tumor was also examined. The data demonstrates that 3SL remarkably reduced the growth of three different tumors, specifically Lewis lung carcinoma, B16F10 melanoma, and CT26 colon cancer, at doses of 0.5 and $1.0 \mathrm{mg} / \mathrm{kg}$, as measured by the volume and weight of tumors (Figure 6A6C). 3SL also inhibited angiogenesis on allograft tumor tissues (Figure 6D). To exclude the possibility that growth inhibition could result from a direct cytotoxic effect, the in vitro cytotoxic effect of 3SL was evaluated. The results demonstrated that $3 \mathrm{SL}$ did not have any significant cytotoxic effect on the cells used for allograft (data not shown).

\section{DISCUSSION}

Angiogenesis, the sprouting of new vessels from existing ones, is one of the hallmarks of cancer [14]. This essential step supports tumor progression by supplying nutrients and oxygen as well as enhancing tumor metastasis by serving as a gateway for entering the vascular system $[15,16]$. VEGF is a primary angiogenic factor in tumor angiogenesis that drives the growth of solid tumors [17]. VEGF secreted from the tumor and stromal cells in the tumor microenvironment increases the growth and migration of vascular endothelial cells as well as the permeability of blood vessels [18]. Although several members of the VEGF family have been identified,
A)
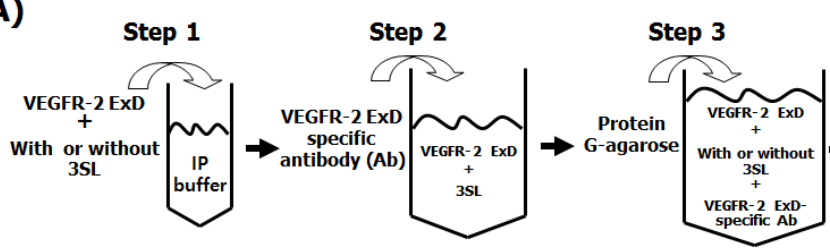

B)

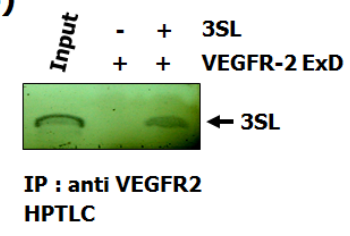

C)

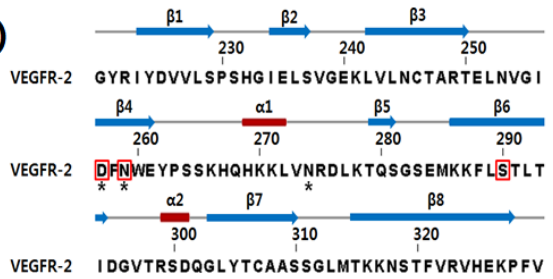

F)

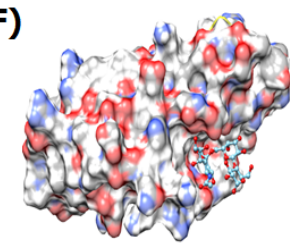

H)

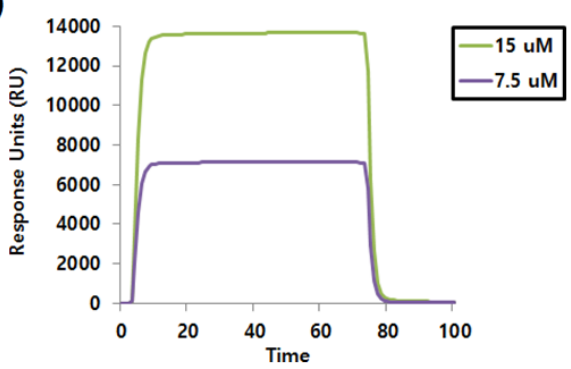

D)

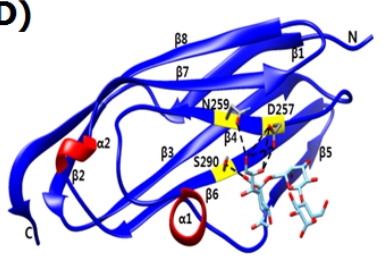

E)

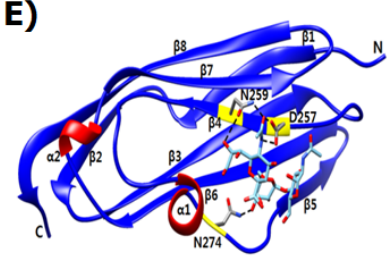

G)

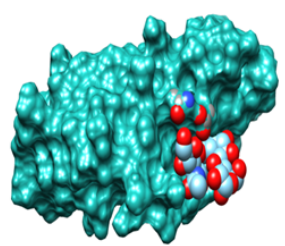

I)

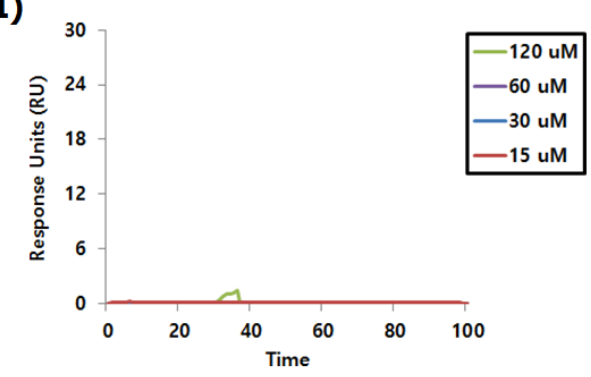

Figure 4: Binding of 3SL to the ExD of VEGFR-2. (A) Schematic presentation of the experimental procedure demonstrating the binding between sialyllactose and the extracellular domain of VEGFR-2. (B) Sialyllactose bound to the ExD of VEGFR-2 and the complex was immunoprecipitated using a VEGFR-2 specific antibody. After washing, sialyllactose was released and analyzed by HPTLC. Lane 1 indicates that the HPTLC loaded with SL as a loading control is developed with a resorcinol-hydrochloric acid reagent. (C) Sequence alignment and the secondary structure of the VEGFR-2 third IgG-like domain are shown. Secondary structure elements are indicated with the arrow ( $\beta$-sheets) and rectangle ( $\alpha$-helix). The linker loops are shown as gray lines. Every ten residues are indicated by a bar. The interaction residues of 3SL and sialyl $N$-actetyllactosamine are indicated by red boxes and black stars, respectively. (D-E) Ribbon representations of the VEGFR-2 structure with 3SL or sialyl $N$-actetyllactosamine are shown. (F) Surface diagrams of VEGFR-2 with 3SL bound at the pocket (left: stick model, right: space filling shaped). Carbon atoms in gray, oxygen atoms in red, nitrogen atoms in blue, and sulfur atom in gold are shown. (G) Surface representation of VEGFR-2 with sialyl $N$-actetyllactosamine is shown. The interaction of (H) 3SL and (I) 3-sialyl $N$-actetyllactosamine with the 2-3 Ig-like domain of VEGFR-2 was measured using the Biocore assay. 
A) con
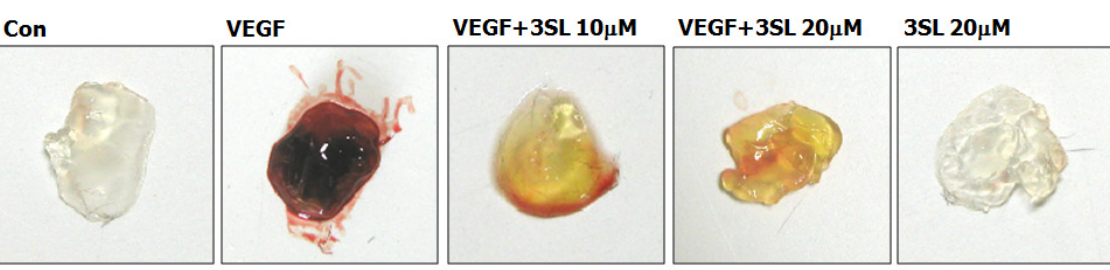

B) Con

VEGF
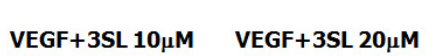

$3 S L 20 \mu M$
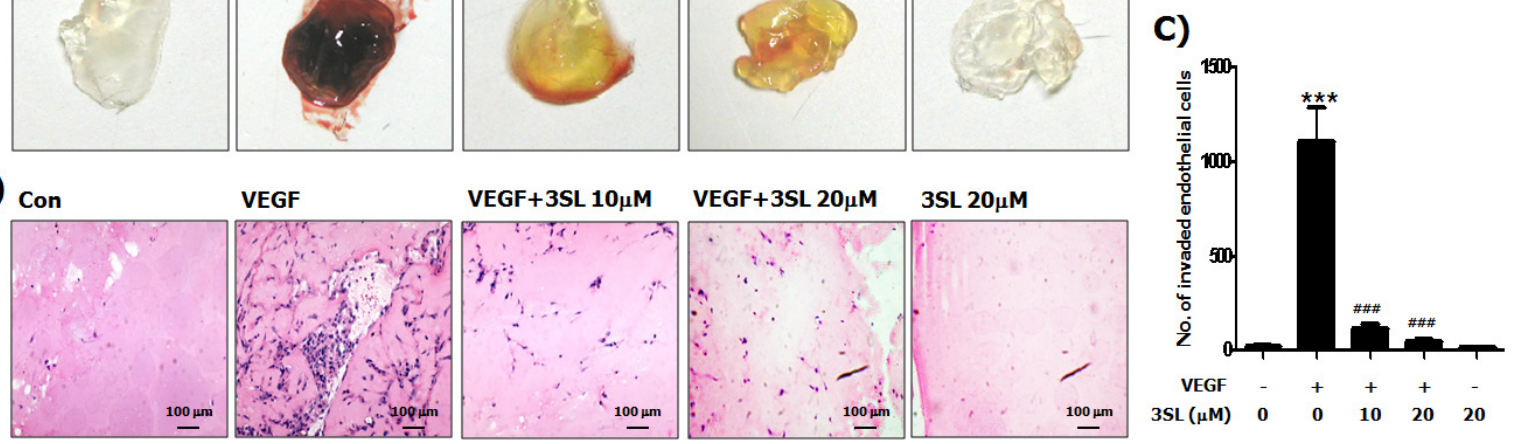

Figure 5: Inhibitory effect of sialyllactose on VEGF-induced in vivo neovascularization. Matrigels were mixed with the indicated concentrations of sialyllactose in the presence of VEGF $(50 \mathrm{ng} / \mathrm{mL})$. The mixed matrigels were inoculated into the abdomen of mice. After a week, the matrigels were separated from euthanized mice. (A) Representative photographs and (B) histological images are shown. (C) Endothelial cells in paraffin section of Matrigel plug were counted. ${ }^{* * *} p<0.001$ compared to the control ( $1^{\text {st }}$ lane), \#\#\# $p<0.001$ compared to the positive control ( $\left.2^{\text {nd }} l a n e\right)$.
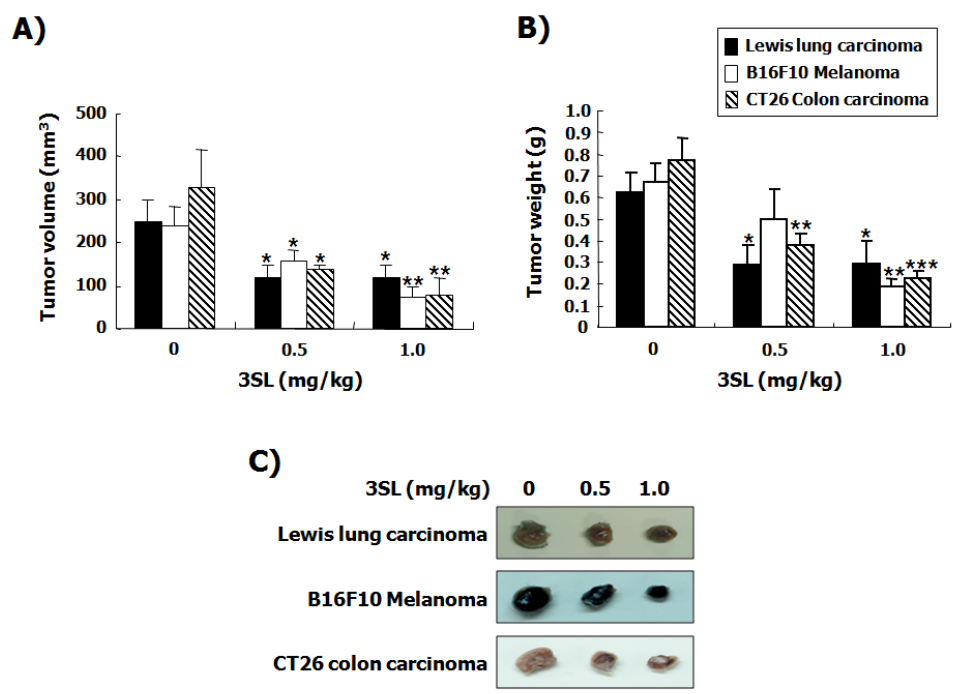

D)
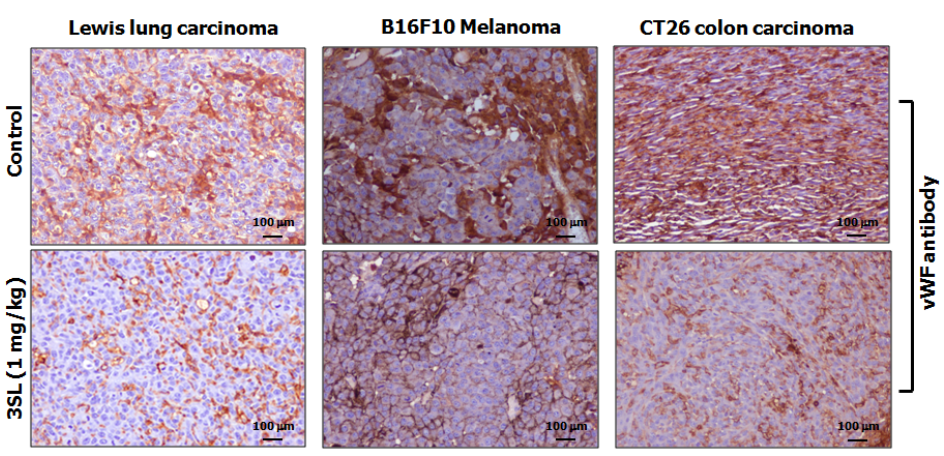

Figure 6: Suppression of tumor growth by sialyllactose in tumor-bearing mice. Lewis lung carcinoma cells $\left(5 \times 10^{5}\right.$ cells $/ 100$ $\mu \mathrm{L}$ in PBS), B16F10 melanoma cells $\left(5 \times 10^{5}\right.$ cells $/ 100 \mu \mathrm{L}$ in PBS), and CT26 colon carcinoma cells $\left(5 \times 10^{5}\right.$ cells $/ 100 \mu \mathrm{L}$ in PBS) were subcutaneously inoculated into the back of mice. Three days after inoculation, the indicated dosages of sialyllactose were intraperitoneally injected into mice daily. Tumor volume (A) and weight (B) were measured at the end of the experiment. The results are presented as the mean \pm SD. ${ }^{*} p<0.05, * * p<0.01$ and ${ }^{* * *} p<0.001$ compared to the control ( $1^{\text {st }}$ lane). (C) Representative images of each tumor treated with the indicated dose of sialyllactose are shown. (D) Tumor tissues were immunostained with the vWF antibody to detect newly formed vessels on tumor tissues in mice inoculated with cancer cells. 
VEGF-A, which is produced by most of cancer cells and upregulated by hypoxia, plays dominant roles in tumor angiogenesis $[15,19,20]$. In addition, of the three different VEGF receptors, VEGFR-2 is the main responder to VEGF-A in in vivo tumor angiogenesis [15].

Binding of VEGF-A to the ExD of VEGFR-2 and homo-dimerization of the receptors can activate subsequent signaling pathways via phosphorylation of the intracellular tyrosine kinase domain of VEGFR-2 $[18,20]$. Here, we demonstrated that sialyllactose inhibits VEGFR-2 phosphorylation and VEGF/VEGFR2-mediated intracellular signaling pathways, including phosphoinositide 3-kinase (PI3K)/Akt, ERK, and p38 in VEGF-stimulated endothelial cells (Figures 1 and 2). In addition, 3SL effectively suppressed VEGF-induced proliferation, tube formation, and migration of vascular endothelial cells (Figures 2 and 3). These results clearly demonstrate that sialyllactose plays an important role in anti-angiogenesis through suppression of VEGF-activated vascular endothelial cells. Moreover, 3SL markedly reduced the formation of VEGF-stimulated new vascular vessels and the growth of allograft tumors in in vivo systems (Figures 5 and 6). VEGF can also enhance the growth of some tumor cells as well as the formation of new vessels in a paracrine or autocrine manner [21]. Therefore, we confirmed whether 3SL also has an effect on tumor growth. The growth of tumor cells, including Lewis lung carcinoma, B16F10 melanoma, and CT26 colon cancer, which were used for the allograft study, were not affected by treatment of 3SL (data not shown). Based on these results, we suggest that the inhibitory effect of 3SL on the growth of allograft tumor cells is mainly due to its anti-angiogenic function.

Inhibition of VEGFR-2 activation can be achieved through diverse molecular mechanisms, such as through an inhibitor or decoy interacting with VEGF, the VEGF binding site of VEGFR-2, or the intracellular tyrosine kinase domain [18, 20-22]. In (Figure 4), we demonstrate that 3SL directly bound to the ExD of VEGFR-2, particularly the third IgG-like domain, a VEGF binding site. To investigate the structural aspects of the VEGFR-2 and 3SL interaction, we used a known third IgG-like domain (amino acids 219-330) structure of human VEGFR-2 (Figure 4D). The third IgG-like domain of VEGFR-2 is composed of eight-stranded $\beta$-sheets with two short $\alpha$-helices (Figure 4C). 3SL interacts strongly with the negatively charged Asp257 and the two polar amino acids Asn259 and Ser290 of VEGFR-2 through the ionic and hydrogen bonds on the concave surface (Figure 4F).

In agreement with a previous study [13], HMOs harboring no sialic acid residue did not affect VEGFinduced phosphorylation of VEGFR-2. However, although sialyllactose is structurally similar to sialyl $\mathrm{N}$ actetyllactosamine, only sialyllactose could suppress the activation of VEGF-mediated VEGFR-2 (Figure 1).
Notably, the addition of an acetamide group to the second carbon position of glucose on sialyllactose hindered the inhibitory effect of sialyllactose on VEGFR-2 phosphorylation induced by VEGF. Similar to 3SL, sialyl $N$-actetyllactosamine interacts with the third IgG-like domain of VEGFR-2 on the concave surface (Figure 4E and 4G). Both sialyl $N$-actetyllactosamine and 3SL molecules bound to the binding pockets surrounded by $\beta 3, \beta 4, \beta 5$, and $\beta 6$ in the third IgG-like domain of VEGFR-2. However, the sialyl $N$-actetyllactosamine interacted with Asn259 and Asp257 polar residues in the pocket as well as with Asn274 at the flexible loop between $\alpha 1$ and $\beta 5$. In this study, the interaction of sialyl $N$-actetyllactosamine with the VEGFR-2 was weaker than that with 3SL. Therefore, we postulated that the sialic acid residue and hydroxyl group on the second carbon of glucose may be essential for inhibiting VEGFR-2 activity. As shown in (Figure 4H and 4I), a Biacore analysis showed that 3SL clearly interacted with the purified second and third IgG-like domains of VEGFR-2, which are well-known VEGF binding sites. However, 3SNL did not. These results suggest that sialyllactose prevents VEGF from binding to VEGFR-2, resulting in the inhibition of VEGF-mediated VEGFR-2 activation and its downstream signaling pathways for inducing anti-angiogenesis.

Several VEGF inhibitors, such as neutralizing VEGF-A antibodies bevacizumab and ranibuzumab, VEGF trapper aflibercept, neutralizing VEGFR-2 antibody ramucirumab, and broad range VEGFR-2 tyrosine kinase inhibitors sunitinib, sorafenid, and pazopanib, are in clinical use or development [15]. However, angiogenesis inhibitors are associated with various side effects, such as hypertension, abnormal bone resorption, and renal toxicity [23-25]. These adverse effects are typical consequences of the suppression of important cellular signaling pathways that regulate and maintain the function of normal tissues [25]. Therefore, the development of selective and safe inhibitors against activation of the VEGF/VEGFR-2 axis is needed. As shown in (Figure 1), as seen with other HMOs, sialyllactose has very low toxicity. However, the selectivity of sialyllactose on VEGFR-2 and its in vivo toxicity should be investigated further.

In conclusion, sialyllactose has an anti-angiogenic property that suppresses the proliferation, tube formation, and migration of vascular endothelial cells through inhibition of VEGF-induced VEGFR-2 activation and consequent signaling pathways via direct interaction with VEGFR-2.

\section{MATERIALS AND METHODS}

\section{Materials}

The oligosaccharides of human milk, such as lactose, lacto $N$-biose, $N$-acetyllactosamine, 3 '-sialyllactosamine, 6 '-sialyllactosamine, $\quad 3$ '-sialyllactose $\quad(3 \mathrm{SL}), \quad$ and 
6'-sialyllactose (6SL), were purchased from Carbosynth Ltd. (Berkshire, UK). Recombinant human vascular endothelial growth factor (VEGF) was supplied by R\&D systems Inc. (Minneapolis, MN, USA). Antibodies against the phosphorylated form or total form of VEGF receptor-2 (VEGFR-2), extracellular signal-regulated kinase (ERK), Akt, and p38 were purchased from Cell Signaling Technology (Danvers, MA, USA). The antibody against von Willebrand Factor (vWF) was purchased from Dako (CA, USA). Antibodies against glyceraldehyde 3-phosphate dehydrogenase (GAPDH) and hoseradish peroxide (HRP)conjugated secondary antibodies were supplied by Santa Cruz Biotechnology (Santa Cruz, CA, USA).

\section{Cell culture}

HUVECs were purchased from Cambrex Inc. (Walkersville, MD, USA) and cultured in sterile endothelial growth medium-2 (EGM-2, Cambrex Inc.). HUVECs at passage number 5 to 8 were used in experiments. Murine lewis lung carcinoma (LLC) cells were purchased from American Type Culture Collection (Manassas, VA, USA). Mouse melanoma (B16F10) and colon carcinoma (CT26) cells were provided by the Korean Cell Line Bank (Seoul, Korea). The cells were cultured with Dulbecco Modified Eagle Medium4 (ThermoFisher Scientific) supplemented with 10\% heatinactivated fetal bovine serum (Sigma-Aldrich, St. Louis, MO, USA), 100 unit $/ \mathrm{mL}$ of penicillin, and $100 \mu \mathrm{g} / \mathrm{mL}$ of streptomycin. All cell lines were maintained at $37^{\circ} \mathrm{C}$ in a humidified $5 \% \mathrm{CO}_{2}$ cell culture incubator.

\section{Cell viability}

The cytotoxic effects of HMOs were examined using the methylthiazolyldiphenyl-tetrazolium bromide assay (MTT, Sigma-Aldrich). In brief, HUVECs were cultured in 24-well plates including endothelial basic medium-2 (EBM-2, Cambrex Inc.) containing 1 \% FBS with the indicated concentration of oligosaccharides in the presence or absence of VEGF (50 ng/mL; R\&D System Inc.) for $72 \mathrm{~h}$. After removal of the culture medium, $300 \mu \mathrm{L}$ of $1 \times$ MTT solution $(0.5 \mathrm{mg} / \mathrm{mL})$ was added to each well. After incubation for $4 \mathrm{~h}$ at $37^{\circ} \mathrm{C}$ in a $\mathrm{CO}_{2}$ incubator, the supernatant was removed, and the formed formazan crystals in viable cells were dissolved in 300 $\mu \mathrm{L}$ of ethanol:DMSO (v/v, 1:1). To detect cytotoxicity, the absorbance in each well was measured at $540 \mathrm{~nm}$ using a SpectraMax M2 reader (Molecular Devices, Sunnyvale, CA, USA). The percentage of living cells in treated cultures was calculated relative to that in untreated cultures.

\section{Western blot analysis}

Total protein from HUVECs treated with the indicated concentration of oligosaccharides in the presence or absence of VEGF was extracted using 1\% NP-40 lysis buffer [150mM NaCl, 10mM HEPES ( $\mathrm{pH}$ 7.45), 1\% NP-40, $5 \mathrm{mM}$ NaPyrophosphate, 5mM NaF, $2 \mathrm{mM} \mathrm{Na} 3 \mathrm{VO} 4]$ containing protease inhibitor cocktail tablet (Roche, Mannheim, Germany). Equal amounts (30 $\mu \mathrm{g})$ of proteins were used for western blot analysis. To detect target proteins, membranes were incubated with a 1:1000 dilution of primary antibodies against each protein, and reacted with the corresponding HRP-conjugated secondary antibodies. Bands were detected with Pierce ECL plus (ThermoFischer Scientific, Waltham, MA, USA) using ImageQuant LAS4000 (GE healthcare, Pittsburgh, PA, USA).

\section{Tube forming assay}

The capillary-like network formation of HUVECs was investigated with Matrigel-coated 24-well culture plates, as previously described [19]. Matrigel (13.9 mg/ $\mathrm{mL}$; BD Bioscience, San Jose, CA, USA) was thawed at $4{ }^{\circ} \mathrm{C}$ and mixed with the same volume of EBM-2 medium. Seventy microliters of the mixture $(6.95 \mathrm{mg} /$ $\mathrm{mL}$ of Matrigel) was added to each well of the 24well culture plates and incubated at $37^{\circ} \mathrm{C}$ for $1 \mathrm{~h}$ for polymerization. The HUVECs were suspended in EBM-2 medium containing $1 \% \mathrm{FBS}\left(1 \times 10^{4}\right.$ cells/well $)$, and were then added to the Matrigel-coated wells at the indicated concentrations of $3 \mathrm{SL}$ in the presence or absence of VEGF. The plates were incubated at $37{ }^{\circ} \mathrm{C}$ in $5 \% \mathrm{CO}^{2}$ atmosphere. After $15 \mathrm{~h}$ incubation, the capillary-like tube formation in each well of the culture plates was photographed with a Nikon Eclipse TS100 microscope (Nikon, Tokyo, Japan).

\section{Migration assay}

The migration assay of HUVECs was performed using 24-well chambers containing polycarbonate filter inserts (Corning Inc., Corning, NY, USA), as previously described [19] with some modification. The HUVECs were suspended in EBM-2 medium containing $1 \% \mathrm{FBS}\left(1 \times 10^{3}\right.$ cells/ $200 \mu \mathrm{L}$ ), and were added to the upper compartment of the chamber with the indicated concentrations of 3SL. EBM-2 medium containing $1 \%$ FBS with or without VEGF was added to the lower chamber. The chambers were incubated at $37{ }^{\circ} \mathrm{C}$ and in $5 \% \mathrm{CO}_{2}$ atmosphere. After $24 \mathrm{~h}$, the HUVECs on the upper side of the filter were removed using cotton swabs. The filters were fixed, stained, and mounted on microscope slides. The migrated HUVECs to the lower side of the filter were counted. The results were calculated as the average of migrated cell numbers from three different filters.

\section{Wound healing assay}

To check migration of unidirectional HUVEC, wound healing assay was performed. The HUVECs 
$\left(5 \times 10^{5}\right.$ cells/well $)$ were seeded in 6-well plates, and cultured in EGM-2 medium. When the cells confluently grew, HUVEC monolayers were scratched with a 10-200 $\mu \mathrm{L}$ pipette tip to make a straight line on the HUVEC monolayer. The cells were treated with or without VEGF in the presence or absence of $3 \mathrm{SL}$, and incubated for 12 $\mathrm{h}$ at $37^{\circ} \mathrm{C}$ and $5 \% \mathrm{CO}_{2}$ atmosphere. Images of the cells were obtained with a Nikon Eclipse TS100 microscope (Nikon, Tokyo, Japan) at $0 \mathrm{~h}$ and $12 \mathrm{~h}$.

\section{Immunofluorescence microscopy}

Immunofluorescence staining was performed as previously described [19]. Briefly, HUVECs were seeded on $12 \mathrm{~mm} \pi$-sterilized coverslips in 24-well tissue culture plates, and treated with the indicated concentration of 3SL in the presence or absence of VEGF. After $24 \mathrm{~h}$, the cells were fixed in $3.7 \%$ formalin, and permeabilized with 0.5 $\%$ Triton X-100 in PBS. After washing three times with PBS, the cells were blocked with PBS containing $1 \%$ bovine serum albumin at room temperature with gentle shaking. Afterwards, a solution (1:50 dilution) of antipaxillin antibody was added to the cells and followed by incubation at $4{ }^{\circ} \mathrm{C}$ overnight. The samples were further incubated with $2 \mu \mathrm{g} / \mathrm{ml}$ of Alexa Fluor 488-conjugated goat anti-rabbit $\mathrm{IgG}$ (Invitrogen) for $1 \mathrm{~h}$ at room temperature. After washing with PBS, the samples were incubated with PBS including 1 unit of Texas Red $囚$-X phalloidin (Invitrogen) for $30 \mathrm{~min}$ at room temperature for actin staining. After washing again with PBS, the samples were analyzed under a fluorescence microscope.

\section{Immunoprecipitation and high performance thin layer chromatography (HPTLC)}

To investigate whether 3SL interacts with VEGFR-2, the purified and identified ExD of VEGFR-2 proteins were used. The VEGFR-2 ExD protein $(1 \mu \mathrm{g})$ was bound with $3 \mathrm{SL}(30 \mu \mathrm{M})$ in $300 \mu \mathrm{L}$ of IP buffer at $4^{\circ} \mathrm{C}$ overnight. The anti-VGFER-2 ExD antibody $(2 \mu \mathrm{g})$ was added to 3SL and VEGFR-2 ExD protein mixtures and incubated at $4^{\circ} \mathrm{C}$ overnight with shaking. The sample was incubated with $20 \mu \mathrm{L}$ of the protein G PLUS-agarose beads (ThermoFisher Scientific) for $4 \mathrm{~h}$ at $4^{\circ} \mathrm{C}$. 3SL interacting with VEGFR-2 ExD was released from the beads through the addition of methanol, and applied to an HPTLC plate with chloroform-methanol- $0.2 \% \mathrm{CaCl}_{2}$ (55:45:10). After separation, 3SL was visualized by spraying the plates with a resorcinol-hydrochloric acid reagent.

\section{Simulation of protein-carbohydrate binding}

Both the 3SL and sialyl $N$-actetyllactosamine interact with ExD of VEGFR-2 (PDB ID: 3S35) as determined by a protein-small molecule docking method. The three-dimensional structure of VEGFR-2 IG3 domain was identified from the RCSB Protein Data Bank and submitted to SwissDock for protein-ligand docking prediction with $3 \mathrm{SL}$ and sialyl $\mathrm{N}$-actetyllactosamine structure information.

\section{Expression and purification of VEGFR-2 IG3}

The vector expressing the second and third IgG-like domain of VEGFR-2 (VEGFR-2 IG3) was constructed according to a previous study [26]. The vector was transformed into overexpression competent cells, Escherichia coli BL21(DE3). Each colony was inoculated in $5 \mathrm{~mL}$ of Luria Bertani (LB) medium enriched with $10 \mu \mathrm{g} / \mathrm{mL}$ kanamycin at $37^{\circ} \mathrm{C}$ overnight. The cells were then incubated in $2 \mathrm{~L}$ of LB containing $10 \mu \mathrm{g} / \mathrm{mL}$ antibiotics at $37^{\circ} \mathrm{C}$ until the $\mathrm{OD}_{600}$ reached 0.5 0.6. Next, the expression of VEGFR-2 IG3 was induced by $0.5 \mathrm{mM}$ isopropyl-thio- $\beta$-d-galactopyranoside (IPTG) at $20^{\circ} \mathrm{C}$ overnight, and the bacterial cells were then harvested by centrifugation at $3,660 \times \mathrm{g}$ for $25 \mathrm{~min}$ at $4^{\circ} \mathrm{C}$. The cell pellets were resuspended in lysis buffer [50 $\mathrm{mM}$ glycine $(\mathrm{pH} 10.5)$ ] containing a protease inhibitor cocktail (Roche Diagnostics), and then sonicated on ice to disrupt the cells using a sonicator (Branson Sonifier 450 sonicator; Danbury, USA). The cell suspensions were centrifuged at $20,170 \times \mathrm{g}$ for $45 \mathrm{~min}$ to separate the supernatant and pellet. Lysis was repeated four times and the final supernatant was concentrated using Vivaspin 20 and centrifuged at $1,320 \times \mathrm{g}$. Finally, the concentrated fractions of VEGFR-2 IG3 were subsequently purified by gel filtration chromatography using a Superdex 200 10/300 GL fast protein liquid chromatography (FPLC) column (GE Healthcare, Sweden) equilibrated in 50 $\mathrm{mM}$ Tris- $\mathrm{HCl}(\mathrm{pH} 8.0)$ and $200 \mathrm{mM} \mathrm{NaCl}$. Concentrated VEGFR-2 IG3 was analyzed by $15 \%$ sodium dodecyl sulfate polyacrylamide gel electrophoresis (SDS-PAGE).

\section{Surface plasmon resonance (SPR) biosensor analysis}

Measurements of the apparent dissociation constants $\left(\mathrm{K}_{\mathrm{D}}\right)$ between VEGFR-2 IG3 and chemical compounds were carried out using a Biacore T100 biosensor (GE Healthcare Biosciences, Sweden). The purified VEGFR-2 IG3 domain was covalently bound to the Series S sensor chip CM5 (carboxylated dextran matrix) using an aminecoupling method, as suggested by the manufacturer. The $150 \mu \mathrm{L}$ of VEGFR-2 $(50 \mu \mathrm{g} / \mathrm{mL})$ in $10 \mathrm{mM}$ sodium acetate pH5.0 was coupled via injection for $15 \mathrm{~min}$ at $10 \mu \mathrm{L} / \mathrm{min}$, followed by injection of $1 \mathrm{M}$ ethanolamine to deactivate the residual amines. For kinetic measurements at $25^{\circ} \mathrm{C}$, chemical compounds with concentrations ranging from 120 to $7.5 \mu \mathrm{M}$ were prepared by dilution in $\mathrm{HBS}^{-\mathrm{EP}^{+}}$ buffer $(10 \mathrm{mM}$ of HEPES, $150 \mathrm{mM}$ of $\mathrm{NaCl}, 3 \mathrm{mM}$ of EDTA, and $0.005 \% \mathrm{v} / \mathrm{v}$ surfactant $\mathrm{P} 20$ ) at a $\mathrm{pH}$ of 7.4 . The immobilized ligand was regenerated by injecting $10 \mu \mathrm{L}$ of $50 \mathrm{mM} \mathrm{NaOH}$ at a rate $10 \mu \mathrm{L} / \mathrm{min}$ during the cycles. 


\section{Animals}

Male C57BL/6 and BALB/c mice (6-7 weeks old, weight 20-22 g), inbred in a specific pathogen-free (SPF) facility, were purchased from Orient Bio, Co. (Seongnam, Korea). The mice were bred separately and had free access to water and a standard diet with a $12 \mathrm{~h}$ light: $12 \mathrm{~h}$ dark cycle. All experimental procedures were examined and approved by the Animal Research Ethics Committee at the Pusan University of Korea.

\section{Matrigel plug assay}

The Matrigel plug assay was performed as previously described [19]. Briefly, C57BL/6 mice were subcutaneously injected with $500 \mu \mathrm{L}$ of a BD Matrigel Matrix and heparin (50 unit $/ \mathrm{mL}$; BD Bioscience) mixture with the indicated concentration of 3SL in presence or absence of VEGF $(100 \mathrm{ng} / \mathrm{mL})$. Five male mice were assigned to each group. After 7 days, the matrigel plugs were removed from the euthanized mice and fixed with $3.7 \%$ formalin in PBS. The plugs were embedded in paraffin, and cut into $4 \mu \mathrm{m}$ sections. The sections were stained with hematoxylin and eosin (H\&E) solutions for microscopic observation.

\section{Tumor allograft}

Each cell line, specifically LLC, B16F10, and CT26, was suspended in PBS $\left(5 \times 10^{5}\right.$ cells $\left./ 100 \mu \mathrm{L}\right)$ and subcutaneously inoculated in the dorsa of 6-7 week-old $\mathrm{C} 57 \mathrm{BL} / 6$ (for LLC and B16F10) and BALB/c mice (for CT26). Six male mice were assigned to each group. Three days after the injection of tumor cells, $3 \mathrm{SL}(0.5$ or $1 \mathrm{mg} / \mathrm{kg}$ in PBS) or PBS was intraperitoneally (i.p.) injected once per day for 12 days. The tumors were excised and weighed 15 days after inoculation of tumor cells. Tumor volumes were measured with a pair of calipers, and were calculated according to the formula [(length $\times$ width $\left.)^{2} / 2\right]$.

\section{Immunohistochemistry}

Tumor specimens were immediately removed from the euthanized tumor-bearing mice, fixed with 3.7 $\%$ formalin in PBS, and then embedded in paraffin for immunohistochemical analysis. The paraffin sections were immunostained with an antibody against the endothelial cell marker vWF, visualized using Dako EnVision kit (Dako, CA, USA), and counterstained with hematoxylin.

\section{Statistical analysis}

The differences between the mean values of experiment groups were determined by one-way analysis of variance (one-way ANOVA) with a Turkey's posthoc test, using GraphPad Prism (Graphpad Software, San Diego, CA, USA). The minimum significance level was set at a $p$ value of 0.05 . All experiments were independently performed at least 3 times.

\section{ACKNOWLEDGMENTS}

This study was financially supported by a National Research Foundation of Korea (NRF) grant from the Ministry of Science, ICT \& Future Planning (MISP), Korea government (Grant no. 2014R1A5A20009936). T.W.C generated most of the data and wrote the draft of the manuscript. E.Y.K, S.J.K, H.J.C, S.H.H, F.A, and C.H.K (Choong-Hwan Kwak) performed experiments. S.B.J performed the simulation and Biacore assay to determine protein-glycan interaction. K.J.K performed histochemical analysis. S.B.J and C.H.K participated in discussion of the results. C.H.K and K.T.H designed this study, supervised the research, and prepared the manuscript.

\section{CONFLICTS OF INTEREST}

The authors have no conflicts of interest.

\section{REFERENCES}

1. Charbonneau MR, O'Donnell D, Blanton LV, Totten SM, Davis JC, Barratt MJ, Cheng J, Guruge J, Talcott M, Bain JR, Muehlbauer MJ, Ilkayeva O, Wu C, et al. Sialylated Milk Oligosaccharides Promote Microbiota-Dependent Growth in Models of Infant Undernutrition. Cell. 2016; 164:859-871.

2. Smilowitz JT, Lebrilla CB, Mills DA, German JB, Freeman SL. Breast milk oligosaccharides: structurefunction relationships in the neonate. Annu Rev Nutr. 2014; 34:143-169.

3. Wu S, Grimm R, German JB, Lebrilla CB. Annotation and structural analysis of sialylated human milk oligosaccharides. J Proteome Res. 2011; 10:856-868.

4. Bode L. Human milk oligosaccharides: every baby needs a sugar mama. Glycobiology. 2012; 22:1147-1162.

5. Newburg DS, Walker WA. Protection of the neonate by the innate immune system of developing gut and of human milk. Pediatr Res. 2007; 61:2-8.

6. Hill DR, Newburg DS. Clinical applications of bioactive milk components. Nutr Rev. 2015; 73:463-476.

7. Bode L, Kunz C, Muhly-Reinholz M, Mayer K, Seeger W, Rudloff S. Inhibition of monocyte, lymphocyte, and neutrophil adhesion to endothelial cells by human milk oligosaccharides. Thromb Haemost. 2004; 92:1402-1410.

8. Idota T, Kawakami H, Murakami Y, Sugawara M. Inhibition of cholera toxin by human milk fractions and sialyllactose. Biosci Biotechnol Biochem. 1995; 59:417-419.

9. Stowell SR, Ju T, Cummings RD. Protein glycosylation in cancer. Annu Rev Pathol. 2015; 10:473-510.

10. Collins PM, Bum-Erdene K, Yu X, Blanchard H. Galectin-3 interactions with glycosphingolipids. Journal of molecular biology. 2014; 426:1439-1451. 
11. Burns AJ, Rowland IR. Anti-carcinogenicity of probiotics and prebiotics. Curr Issues Intest Microbiol. 2000; 1:13-24.

12. Kuntz S, Rudloff S, Kunz C. Oligosaccharides from human milk influence growth-related characteristics of intestinally transformed and non-transformed intestinal cells. Br J Nutr. 2008; 99:462-471.

13. Rudloff S, Schneider D, Kunz C, Bretzel RG, Linn T. Human milk as a natural source of anti-angiogenic compounds. Pediatr Res. 2004; 56:503-503.

14. Hanahan D, Weinberg RA. Hallmarks of cancer: the next generation. Cell. 2011; 144:646-674.

15. Claesson-Welsh L, Welsh M. VEGFA and tumour angiogenesis. J Intern Med. 2013; 273:114-127.

16. Paduch $\mathrm{R}$. The role of lymphangiogenesis and angiogenesis in tumor metastasis. Cell Oncol (Dordr). 2016.

17. Abraham AG, O'Neill E. PI3K/Akt-mediated regulation of p53 in cancer. Biochemical Society transactions. 2014; 42:798-803.

18. Ferrara N. Vascular endothelial growth factor: basic science and clinical progress. Endocr Rev. 2004; 25:581-611.

19. Chung TW, Kim SJ, Choi HJ, Kwak CH, Song KH, Suh SJ, Kim KJ, Ha KT, Park YG, Chang YC, Chang HW, Lee YC, Kim CH. CAPE suppresses VEGFR-2 activation, and tumor neovascularization and growth. Journal of molecular medicine. 2013; 91:271-282.
20. Ferrara N. Pathways mediating VEGF-independent tumor angiogenesis. Cytokine Growth Factor Rev. 2010; 21:21-26.

21. Roskoski R Jr. Vascular endothelial growth factor (VEGF) signaling in tumor progression. Crit Rev Oncol Hematol. 2007; 62:179-213.

22. Limaverde-Sousa G, Sternberg C, Ferreira CG. Antiangiogenesis beyond VEGF inhibition: a journey from antiangiogenic single-target to broad-spectrum agents. Cancer Treat Rev. 2014; 40:548-557.

23. Alameddine RS, Yakan AS, Skouri H, Mukherji D, Temraz S, Shamseddine A. Cardiac and vascular toxicities of angiogenesis inhibitors: The other side of the coin. Crit Rev Oncol Hematol. 2015; 96:195-205.

24. Aleman JO, Farooki A, Girotra M. Effects of tyrosine kinase inhibition on bone metabolism: untargeted consequences of targeted therapies. Endocr Relat Cancer. 2014; 21:R247-259.

25. Kamba T, McDonald DM. Mechanisms of adverse effects of anti-VEGF therapy for cancer. Br J Cancer. 2007; 96:1788-1795.

26. Fuh G, Li B, Crowley C, Cunningham B, Wells JA. Requirements for binding and signaling of the kinase domain receptor for vascular endothelial growth factor. J Biol Chem. 1998; 273:11197-11204. 